\title{
Strong Correlations between Sensitivity and Variability Give Rise to Constant Discrimination Thresholds across the Otolith Afferent Population
}

\author{
Mohsen Jamali, Jerome Carriot, Maurice J. Chacron, and Kathleen E. Cullen \\ Department of Physiology, Aerospace Medical Research Unit, McIntyre Medical Building, McGill University, Montreal, Quebec H3G 1Y6, Canada
}

The vestibular system is vital for our sense of linear self-motion. At the earliest processing stages, the otolith afferents of the vestibular nerve encode linear motion. Their resting discharge regularity has long been known to span a wide range, suggesting an important role in sensory coding, yet to date, the question of how this regularity alters the coding of translational motion is not fully understood. Here, we recorded from single otolith afferents in macaque monkeys during linear motion along the preferred directional axis of each afferent over a wide range of frequencies $(0.5-16 \mathrm{~Hz})$ corresponding to physiologically relevant stimulation. We used signal-detection theory to directly measure neuronal thresholds and found that values for single afferents were substantially higher than those observed for human perception even when a Kaiser filter was used to provide an estimate of firing rate. Surprisingly, we further found that neuronal thresholds were independent of both stimulus frequency and resting discharge regularity. This was because increases in trial-to-trial variability were matched by increases in sensitivity such that their ratio remains constant: a coding strategy that markedly differs from that used by semicircular canal vestibular afferents to encode rotations. Finally, using Fisher information, we show that pooling the activities of multiple otolith afferents gives rise to neural thresholds comparable with those measured for perception. Together, our results strongly suggest that higher-order structures integrate inputs across afferent populations to provide our sense of linear motion and provide unexpected insight into the influence of variability on sensory encoding.

\section{Introduction}

As we navigate through the world, our brain integrates information originating from the vestibular, visual, and proprioceptive systems to compute an estimate of self-motion. However, vestibular contributions to motion perception have been difficult to study because the pathways are inherently multisensory. Numerous studies have attempted to quantify perceptual vestibular thresholds for linear motion in humans (Walsh, 1961, 1962; Young and Meiry, 1968; Jones and Young, 1978; Benson et al., 1986; Zupan and Merfeld, 2008; MacNeilage et al., 2010a,b; Naseri and Grant, 2012). Notably, recent results show that vestibular cues play a central role in determining perceptual thresholds and further suggest that they can be as low as $\sim 1.5 \mathrm{~cm} / \mathrm{s}^{2}$ (Valko et al., 2012).

To date, the neural mechanisms that give rise to our sense of linear self-motion are essentially unknown. At the earliest stages of vestibular processing, otolith afferents detect linear acceleration. Studies performed in other systems show that central pathways must integrate information transmitted by peripheral sensory neuron populations to explain perceptual thresholds as peripheral sensory neurons display much higher values (visual:

\footnotetext{
Received Jan. 30, 2013; revised May 15, 2013; accepted June 3, 2013.

Author contributions: M.J. and K.E.C. designed research; M.J. and J.C. performed research; M.J. analyzed data; M.J., M.J.C., and K.E.C. wrote the paper.

We thank S. Sadeghi for contributing to data acquisition and S. Nuara and W. Kucharski for technical assistance.

Correspondence should be addressed to Kathleen E. Cullen, Aerospace Medical Research Unit, 3655 Drummond Street, Montreal, QC H3G 1Y6, Canada. E-mail: kathleen.cullen@mcgill.ca.

DOI:10.1523/JNEUROSCI.0459-13.2013

Copyright $\odot 2013$ the authors $\quad 0270-6474 / 13 / 3311302-12 \$ 15.00 / 0$
}

Pelli, 1985; Amano et al., 2006; Shadlen et al., 1996; auditory: Pfingst and Xu, 2004; Bizley et al., 2010; cross modal: McDonald et al., 2000; Shams et al., 2000, 2002; vestibular (rotation): Sadeghi et al., 2007; Massot et al., 2011). Surprisingly, however, Yu et al. (2012) recently extrapolated threshold values for single macaque otolith afferents that were comparable with perceptual ones, which suggests that the vestibular system uses a unique strategy for coding linear acceleration.

Here we address the important question of whether the otolith system uses a fundamentally different strategy than other senses. To do so, we recorded single otolith afferent activity in macaque monkeys during linear motion along the axis for which each individual afferent displayed maximal sensitivity. We then used signal-detection theory to directly measure neural thresholds. Specifically, to understand how the vestibular periphery encodes linear motion during everyday activities, neural thresholds were computed for frequencies $(0.5-16 \mathrm{~Hz})$ that span the physiologically relevant range. First, we found that neuronal threshold values for single afferents are substantially higher than reported perceptual threshold values. Second, although otolith afferents can be segregated into two functional groups (regular and irregular) based on resting discharge regularity (for review, see Goldberg, 2000), we establish that resting discharge regularity has no influence on neural thresholds because threshold values were not significantly different between regular and irregular afferents. Indeed, our analysis demonstrates that increases in trial-to-trial variability are matched by increases in response sensitivity such that their ratio remains constant, which effectively overcomes its 


\section{A Recording site}

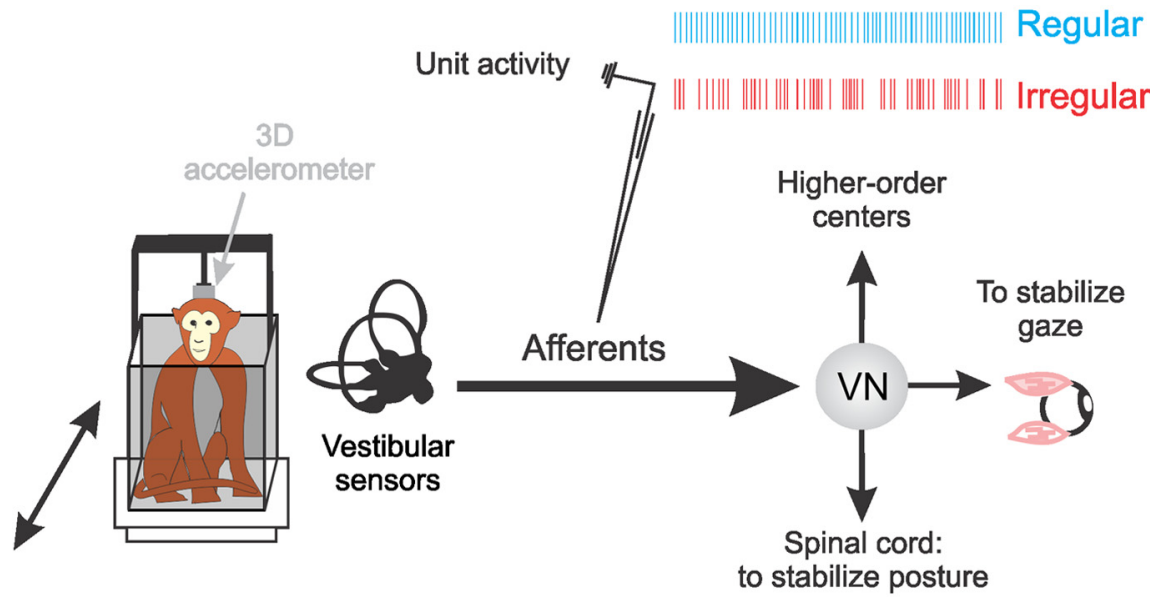

B Afferent's preferred direction
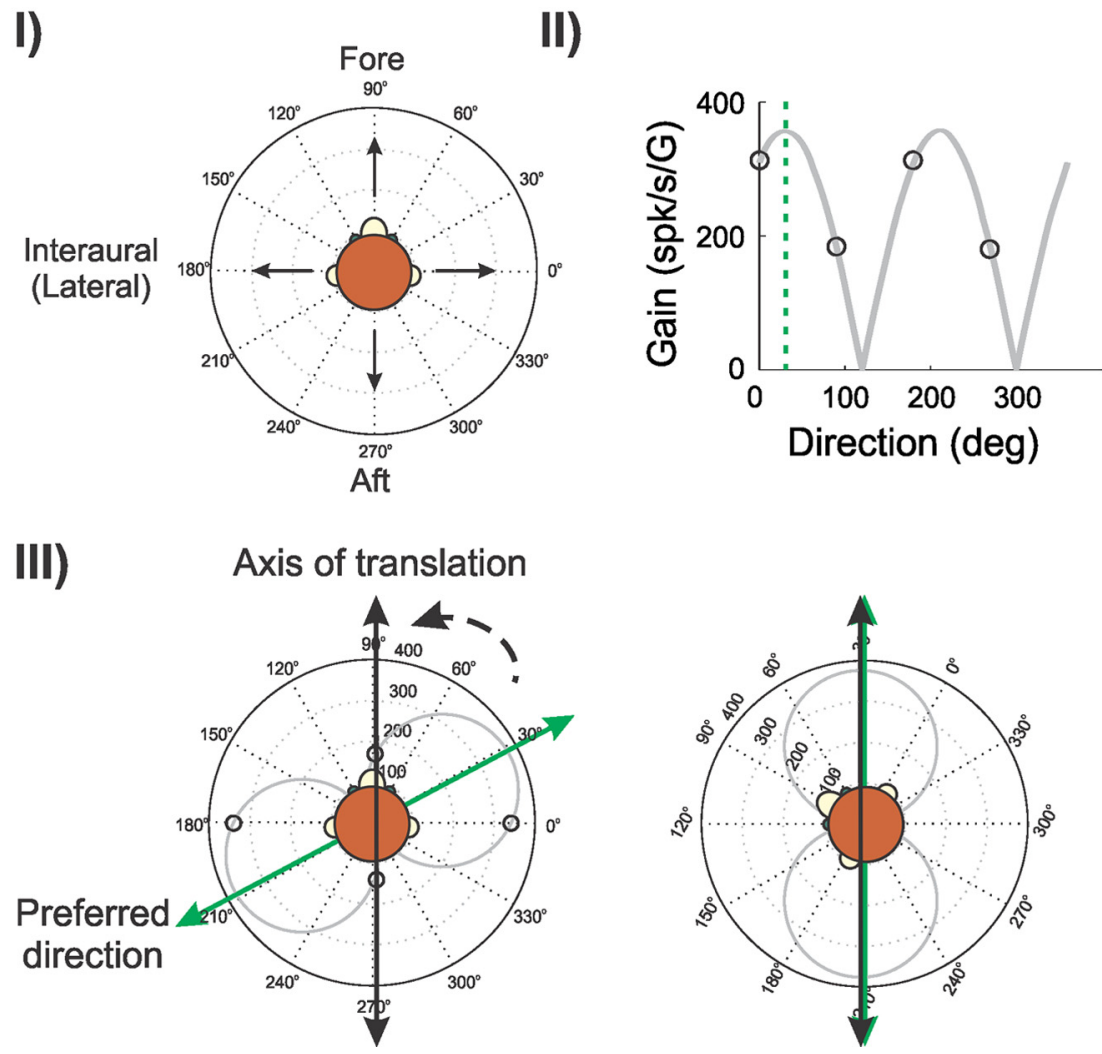

Figure 1. Experimental procedures. $\boldsymbol{A}$, We recorded extracellular single-unit activity from otolith afferents using glass microelectrodes. We measured head acceleration using a three-dimensional linear accelerometer. VN, Vestibular nuclei. $\boldsymbol{B}$, We determined the PD of each afferent in the following way. First, we applied sinusoidal translation $(5 \mathrm{~Hz}, 0.2 \mathrm{G})$ along the fore-aft and lateral directions in the horizontal plane ( $\boldsymbol{I}$ ) and estimated sensitivity in each direction (II, black circles). Second, the sensitivity for an arbitrary direction was estimated by a cosine fit (II, gray curve), and the PD was defined as the direction for which sensitivity was maximal (II, dashed green line). We stimulated each afferent along its PD by rotating the monkey's head (III, dashed arrow) to align the axis of translation (III, black arrow) with PD (III, green arrow).

detrimental effects. Finally, using Fisher information (FI), we provide a lower-bound estimate for the otolith afferent population size over which information needs to be integrated to give rise to perceptual thresholds. We conclude that the otolith system does not use a fundamentally different strategy than other senses to give rise to perceptual thresholds. Moreover, our findings have important im- plications regarding the role of neural variability on sensory coding and the limitations that it imposes on sensory performance.

\section{Materials and Methods}

Three male macaque monkeys (Macaca fascicularis) were prepared for chronic extracellular recording using aseptic surgical techniques. All procedures were approved by the McGill University Animal Care Committee and were in compliance with the guidelines of the Canadian Council on Animal Care.

\section{Surgical procedures}

The surgical preparation was similar to that described previously (Dale and Cullen, 2013). Briefly, using aseptic surgical techniques and under isoflurane anesthesia $(0.8-1.5 \%)$, a stainless steel post was secured to the animal's skull with stainless steel screws and dental acrylic resin, allowing complete immobilization of the head during the experiments. The implant also held in place a recording chamber oriented stereotaxically toward the vestibular nerve where it emerges from the internal auditory meatus. Finally, an 18to 19-mm-diameter eye coil (three loops of Teflon-coated stainless steel wire) was implanted in the right eye behind the conjunctiva. After the surgery, buprenorphine $(0.01 \mathrm{mg} / \mathrm{kg}$, i.m.) was administered as postoperative analgesia every $12 \mathrm{~h}$ for $2-5 \mathrm{~d}$ depending on the animal's pain level, and Anafen $(2 \mathrm{mg} / \mathrm{kg}$ and then $1 \mathrm{mg} / \mathrm{kg}$ on subsequent days) was used as an antiinflammatory. In addition, cefazolin $(25 \mathrm{mg} / \mathrm{kg}$, i.m.) was injected twice daily for $10 \mathrm{~d}$. Animals were given at least 2 weeks to recuperate from the surgery before any experiments began.

\section{Data acquisition}

During experiments, the head-restrained monkey was seated in a primate chair mounted on top of a vestibular turntable in a dimly lit room. The vestibular nerve was approached through the floccular lobe of the cerebellum, as identified by its eye-movement-related activity (Lisberger and Pavelko, 1986; Cullen and Minor, 2002); entry to the nerve was preceded by a silence, indicating that the electrode had left the cerebellum. Extracellular single-unit activity of otolith afferents was recorded using glass microelectrodes (24-27 $\mathrm{M} \Omega$ ) as described previously (Jamali et al., 2009). Head acceleration was measured by a threedimensional linear accelerometer (ADXL330Z; Analog Devices) firmly secured to the animal's head post (Fig. 1A). During experimental sessions, unit activity, horizontal and vertical eye positions, and head acceleration signals were recorded on digital audiotape for later playback. During playback, action potentials from extracellular recordings were discriminated using a windowing circuit (BAK Electronics). Eye position and head acceleration signals were low-pass filtered at $250 \mathrm{~Hz}$ (eight-pole Bessel filter) and sampled at $1 \mathrm{kHz}$. Data were imported into MATLAB (MathWorks) for analysis using custom-written algorithms.

\section{Experimental design}

All afferents that were included in the present study responded to sinusoidal translational head movements $(5 \mathrm{~Hz}, 0.2 \mathrm{G})$ applied along the 
fore-aft $\left(90^{\circ}\right)$ and/or lateral $\left(0^{\circ}\right)$ axes (Fig. $\left.1 B I\right)$ and did not respond to yaw rotations. Because of limitations of our experimental setup, afferents that were predominantly sensitive to stimulation along the vertical axis were not included in our dataset.

Preferred direction (on-line computation) of afferents. Once an afferent fiber was isolated, we first determined the axis along which the fiber is maximally responsive. We henceforth refer to this axis as the preferred direction (PD). PD was determined using the following methodology. First, we applied sinusoidal translation $(5 \mathrm{~Hz}, 0.2 \mathrm{G})$ along the fore-aft and lateral axes in the horizontal plane (Fig. $1 B I$ ), and the sensitivity of the unit was computed (described below) in both directions (Fig. 1BII) using Spike2 software (Cambridge Electronics Design) and customwritten MATLAB algorithms. We then used these measurements to estimate the tuning curve (i.e., the sensitivity as a function of direction) using a cosine fit (Fig. 1BII) (Fernández and Goldberg, 1976a; Angelaki and Dickman, 2000; Purcell et al., 2003). The PD was taken as the direction for which sensitivity was maximal.

Vestibular stimulation. All subsequent stimulation was applied along PD (Fig 1BIII). The stimulation to the afferent consisted of sinusoidal translations at frequencies of $0.5,1,2,3,4,5,6,7,8,9,10,11,12,13,14$, 15 , and $16 \mathrm{~Hz}$ with peak acceleration at $0.2 \mathrm{G}\left(196 \mathrm{~cm} / \mathrm{s}^{2}\right)$ for all frequencies except $0.5 \mathrm{~Hz}$. Note that, for $0.5 \mathrm{~Hz}$ stimulation, the peak acceleration was either 0.15 or $0.1 \mathrm{G}$ (147 or $98 \mathrm{~cm} / \mathrm{s}^{2}$, respectively).

\section{Analysis of neuronal discharges}

Background discharge. Otolith afferents are characterized as either regular or irregular based on their resting discharge, which is correlated with anatomical differences in location and peripheral terminations (for review, see Goldberg, 2000; Eatock and Songer, 2011). Regularity of resting discharge was measured by means of coefficient of variation $(\mathrm{CV})$, $\mathrm{CV}=\frac{\mu_{\mathrm{ISI}}}{\sigma_{\mathrm{ISI}}}$, where $\mu_{\mathrm{ISI}}$ and $\sigma_{\mathrm{ISI}}$ are the mean and SD of the interspike intervals (ISIs) recorded during spontaneous background activity. Because CV varies with the mean ISI, a normalized coefficient of variation $\left(\mathrm{CV}^{\star}\right)$ was computed using the ISI distribution to quantify resting discharge regularity following the same procedure used previously by Goldberg et al. (1984). Afferents with $\mathrm{CV}^{\star}<0.1$ were classified as regular, whereas those with $\mathrm{CV}^{\star} \geq 0.1$ were classified as irregular (Goldberg et al., 1990; Jamali et al., 2009; Yu et al., 2012).

Firing rate estimation. The time-dependent firing rate $f r(t)$ evoked by sinusoidal stimulation along the PD of the afferent was estimated in two ways. First, the spike train $r(t)$ was represented as a binary sequence with bin width of $1 \mathrm{~ms}$. The value of bin $j$ was set to 1 if it contained an action potential and 0 otherwise as described previously (Sadeghi et al., 2007). Then, $f r(t)$ was obtained by convolving the spike train $r(t)$ with a Kaiser window whose cutoff frequency was set to $0.1 \mathrm{~Hz}$ above the stimulus frequency to ensure that the pass band of the filter includes the frequency of the stimulus (Cherif et al., 2008; Jamali et al., 2009; Yu et al., 2012). Alternatively, we estimated the time-dependent firing rate $f r_{\text {ISI }}(t)$ as the reciprocal of the ISI as described previously (Yu et al., 2012).

We chose the Kaiser filter and reciprocal ISI to estimate the instantaneous firing rate because they can be viewed as two examples of estimating firing rate from spike trains that are on the opposite ends of the spectrum: (1) extensive filtering using a Kaiser window and (2) no filtering by using reciprocal ISI. Notably, it has been shown that Kaiser filters consistently outperformed currently used methods, such as reciprocal ISI (and also spike density function and rate histogram) particularly at higher frequencies, as characterized by minimal aliasing, phase, and amplitude distortion (Cherif et al., 2008). Thus, using a Kaiser filter gives rise to lower threshold values than other techniques (Yu et al., 2012). Therefore, we used a Kaiser filter to obtain a lower bound on the estimates of neuronal thresholds. However, to quantify the effect of filtering on the threshold value obtained, we compared threshold estimates using the Kaiser filter to those obtained using the reciprocal ISI.

Response dynamics. At each frequency, response sensitivity and phase were estimated by fitting a first-order model to the data:

$$
\hat{f r}(t)=b+\text { Gain } \times \ddot{\mathrm{H}}\left(\mathrm{t}-\mathrm{t}_{d}\right),
$$

where $\hat{f r}(t)$, is the predicted firing rate, $b$ is a bias term representing the resting discharge rate, Gain is the response sensitivity, $\ddot{H}(t)$ is the linear head acceleration, and $t_{d}$ is the dynamic lead time. We used a leastsquares regression to find parameter values that maximize the varianceaccounted-for (VAF) given by the following:

$$
\mathrm{VAF}=1-\left[\frac{\operatorname{var}(\hat{f r}(t)-f r(t)}{\operatorname{var}(f r(t))}\right],
$$

where var is the variance (Cullen et al., 1996).

Consistent with previous studies (Fernández and Goldberg, 1976a), we found a positive correlation between the resting discharge rate and sensitivity for regular afferents $\left(R^{2}=0.67, p=0.0002\right)$ but not for irregular afferents $\left(R^{2}=0.01, p=0.71\right)$.

Neuronal threshold calculation. To compute the neuronal threshold of an individual otolith afferent for a given stimulus frequency, we first plotted its time-dependent firing rate as a function of the shifted stimulus $\ddot{H}\left(t-t_{d}\right)$ to obtain the instantaneous firing rate-head acceleration curve (see Fig. $3 A$, top and bottom). Next, using an acceleration bin width of $10 \mathrm{~cm} / \mathrm{s}^{2}$, we computed the mean and variance of the corresponding firing rate distribution for each head acceleration value (see Fig. $3 A$, bottom). The neuronal thresholds were computed using different methodologies. The first method used the $d^{\prime}$ measure from signal-detection theory (Green and Swets, 1966), which assumes that the firing rate distribution is normal:

$$
d^{\prime}(\ddot{H})=\frac{|\mu(\ddot{H})-\mu(0)|}{\sqrt{\left(\sigma^{2}(\ddot{H})+\sigma^{2}(0)\right) / 2}},
$$

where $\mu(\ddot{H})$ and $\sigma^{2}(\ddot{H})$ are the mean and variance of the firing rate distribution at head acceleration $\ddot{H}$, and $\mu(0)$ and $\sigma^{2}(0)$ are the mean and variance of the firing rate distribution at zero acceleration, respectively. The $d^{\prime}$ values were then plotted as a function of $\ddot{H}$ and fitted with a first-order polynomial (see Fig. $3 A$, middle). The detection thresholds $T_{d^{\prime} \text { exc }}$ and $T_{d^{\prime} \text { inh }}$ were computed as the absolute value of the positive and negative values of head acceleration for which $d^{\prime}=1$, respectively ( $\mathrm{Sa}$ deghi et al., 2007). Furthermore, the detection threshold value $T_{d^{\prime}}$ was set to the minimum of the two.

The second method used receiver operating characteristic (ROC) analysis. Similar to $d^{\prime}$ measure, we first plotted the time-dependent firing rate of the afferent as a function of the shifted stimulus $\ddot{H}\left(t-t_{d}\right)$ (see Fig. 4A). Next, using an acceleration bin width of $10 \mathrm{~cm} / \mathrm{s}^{2}$, we determined the probability that an ideal observer can distinguish an arbitrary non-zero acceleration $\ddot{H}$ from null using their corresponding empirical firing rate distributions. Specifically, distributions of firing rates and corresponding ROC curves (Green and Swets, 1966) were generated for each value of head acceleration $\ddot{H}$. Then, the probability of correct detection was determined as the area under the ROC curve and was plotted as a function of $\ddot{H}$ to obtain the neurometric curve. Finally, we fit the neurometric curve using an error function (see Fig. $4 A$, inset), and the acceleration detection threshold $T_{\max }$ was defined as the value of head acceleration for which the probability of correct detection was equal to $84 \%$. This approach has the advantage that there is no assumption on the shape of the firing rate distribution.

For the third method, using ROC analysis, we computed discrimination threshold values, $T_{\min -\max }$ as the smallest positive value of acceleration that enables an ideal observer to discriminate between the firing rate distributions obtained for that acceleration and its opposite value with an $84 \%$ probability of correct detection (see Fig. $4 B$ ).

Comparison between threshold estimates using different methodologies. To derive theoretical predictions that allow us to directly compare estimates obtained using different methodologies, we worked under the assumptions that, for a given stimulus, the firing rate variance is independent of head acceleration, the mean firing rate is a linear function of head acceleration, and the firing rate distribution for a given value of head acceleration is Gaussian. We empirically found that these assumptions were valid for our dataset because the firing rate variance at zero acceleration was not significantly different from that at threshold (paired $t$ test with Bonferroni's correction, $p>0.07$ for both Kaiser and 1/ISI). Specifically, the different threshold measures are related by the following (Merfeld, 2011): 


\section{A Example otolith afferents}

\section{Head acceleration}

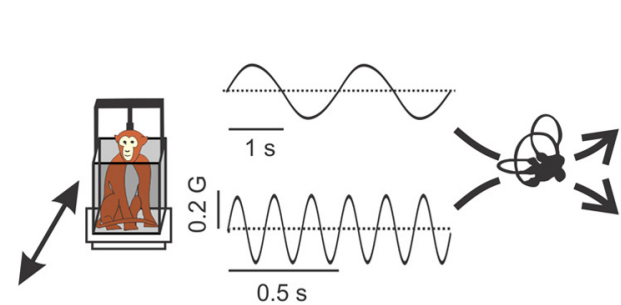

\section{B Population responses}

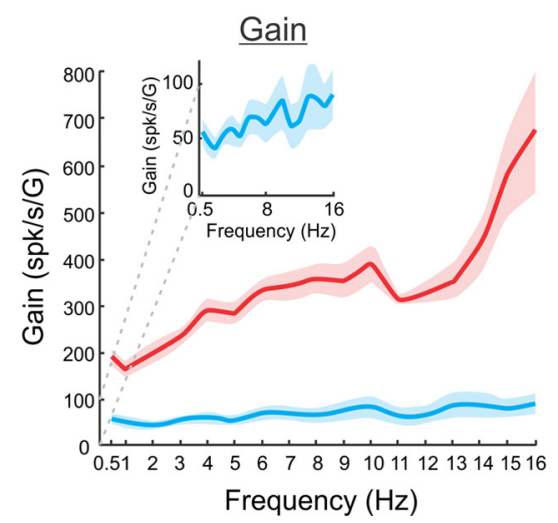

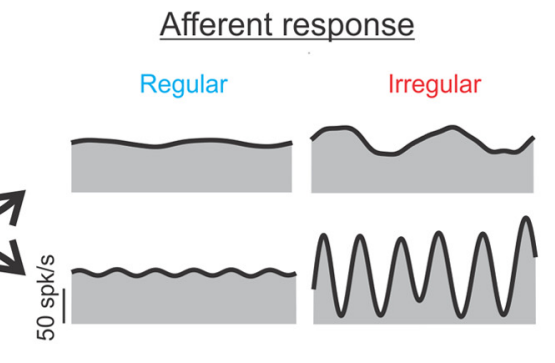

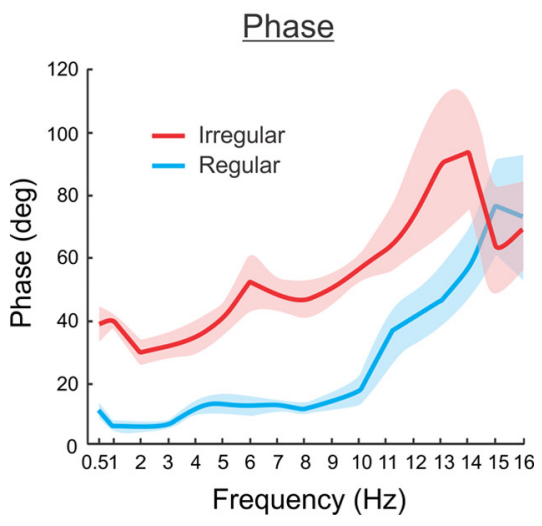

Figure 2. Regular and irregular otolith afferents respond differentially to sinusoidal stimuli. $\boldsymbol{A}$, Time-dependent firing rate (gray) from typical regular (left) and irregular (right) afferents in response to $0.5 \mathrm{~Hz}$ (top) and $6 \mathrm{~Hz}$ (bottom) sinusoidal linear acceleration stimuli. Superimposed are the firing rate estimates based on Equation 1 (solid black trace). B, Population-averaged neuronal gain (i.e., sensitivity) (left) and phase (right) for regular (blue) and irregular (red) otolith afferents as a function of the stimulus frequency. Note that gain increases with stimulus frequency for both afferent types. However, this increase was more pronounced for irregular afferents. The inset expands the $y$-axis to illustrate that the gain of regular afferents also increases as a function of frequency. The shaded bands show 1 SEM.

$$
T_{d^{\prime}}=T_{\max } / \sqrt{2}=\sqrt{2} \times T_{\min -\max } .
$$

Predicting neuronal thresholds based on trial-to-trial variability and sensitivity. Under the assumptions described above and that the model described by Equation 1 is valid, Equation 3 then simplifies to $d^{\prime}(\ddot{H})=\frac{|\mu(\ddot{H})-\mu(0)|}{\sigma(0)}$. Using the relationship $\mu(\ddot{H})=b+$ Gain $\ddot{H}$ (Eq. 1) then gives $d^{\prime}(\ddot{H})=\frac{\text { Gain } \times|\ddot{H}|}{\sigma(0)}$. Therefore, the discrimination threshold measure $T_{\min -\max }$ is predicted to be equal to the following:

$$
T_{\text {pred }}=\frac{\sigma(0)}{\sqrt{2} \text { Gain }},
$$

where $\sigma(0)$ is the SD of the firing rate distribution at zero acceleration. This equation predicts that scaling both the response gain and trial-totrial variability by the same factor will not alter the discrimination threshold.

\section{Population thresholds: Fisher information}

Because perceptual threshold values most likely arise from integration of activities of afferent populations, we used Fisher information (FI; Lehmann, 1983; Gordon et al., 2008) to combine the activities of multiple afferents responding to the same sinusoidal stimuli with a given frequency $f$ to compute threshold values. FI provides a measure to quantify the discriminability of an individual afferent or a population of afferents when the head acceleration varies by a small amount. If we assume that (1) each otolith afferent acts as an individual channel of information (i.e., independent noise among otolith afferents; see Discussion) and (2) the response variance is much smaller than the mean and has a Gaussian distribution, then the contribution of each unit to the FI at any given acceleration is proportional to the square of the slope of the curve relating firing rate to head acceleration divided by the variance of the response at that particular acceleration (Gordon et al., 2008; Gu et al., 2010). Therefore, the population FI is given by the sum of Fisher information of every individual neuron $\left(\mathrm{FI}_{i}\right)$ :

$$
\mathrm{FI}=\sum_{i=1}^{N} \mathrm{FI}_{i}=\sum_{i=1}^{N}\left(\frac{\left|f r_{i}^{\prime}\right|^{2}}{\sigma_{i}^{2}}\right)
$$

where $f r_{i}^{\prime}$ and $\sigma_{i}^{2}$ are the slope and the variance of the firing rate acceleration curve of neuron $i$ at null acceleration, respectively, and $N$ is the population size. The FI provides an upper bound for the discriminability $\left(d^{\prime}\right)$ of a small change in the head acceleration $(\Delta I)$ around zero such that:

$$
d^{\prime} \leq \Delta I \times \sqrt{\mathrm{FI}} .
$$

We used $d^{\prime}=\sqrt{2} / 2$ as the discriminability criterion value to estimate discrimination thresholds (see above) that can then be better compared with perceptual values (see Discussion). To estimate the FI for a population size of $1 \leq N \leq 20$, we randomly sampled up to 500 possible neuronal subgroups of size $N$ without repetition from our neuronal population. For each subgroup, we computed the total FI and estimated the corresponding threshold. The threshold values were then averaged across subgroups.

All values are expressed as mean \pm SEM unless otherwise stated. Statistical significance was set at $p<0.05$. To account for multiple comparisons, a Bonferroni's correction was applied whenever applicable.

\section{Results}

We first recorded the spiking activities of each isolated otolith fiber $(N=76)$ in the absence of stimulation. $N=39$ fibers were classified as regular $\left(\mathrm{CV}^{\star}=0.02-0.09\right)$ and the rest $(N=37)$ were classified as irregular $\left(\mathrm{CV}^{\star}=0.11-0.57\right)$. The average resting rate was similar between the two groups of afferent (regular, $78 \pm 4$ spikes/s; irregular, $79 \pm 5$ spikes/s; $p=0.85$ ). Next, we examined the response characteristics of each individual otolith fiber by stimulating the afferent along its PD using a set of linear acceleration frequencies $(0.5-16 \mathrm{~Hz})$ spanning the physiologically relevant range. Figure $2 A$ shows the time-dependent firing rates of example regular and irregular afferents in response to 0.5 (top traces) and $6 \mathrm{~Hz}$ (bottom traces) sinusoidal stimulation. The responses were consistent with previous studies (Fernández and Goldberg, 1976b; Angelaki and Dickman, 2000) in that (1) the regular afferent displayed lower sensitivity than the irregular afferent for both stimulus frequencies $(0.5 \mathrm{~Hz}$ : 31.5 vs 130.0 spikes $\cdot \mathrm{s}^{-1} \cdot \mathrm{G}^{-1} ; 5 \mathrm{~Hz}$ : 33.1 vs 374.2 spikes $\cdot \mathrm{s}^{-1} \cdot \mathrm{G}^{-1}$ for regular and irregular afferents, respectively), and (2) the irregular afferent displayed a larger increase (approximately three times) in sensitivity as a function of frequency than the regular afferent.

These results were consistently seen across our dataset. On average, irregular afferents displayed larger sensitivity and phase leads than regular afferents (Fig. 2B). Moreover, although the sensitivity of both populations increased as a function of frequency, the rate of increase was greater for irregular afferents (Fig. 2, compare $B$ with $B$, inset). Furthermore, our data indicate that the phase lead increases as a function of frequency for both 
regular and irregular afferent populations (Fig. 2B). Indeed, regular afferents respond nearly in-phase with head acceleration for frequencies up to $10 \mathrm{~Hz}\left(\sim 15^{\circ}\right)$ but display larger phase leads at higher frequencies $\left(\sim 70^{\circ}\right.$ for $\left.16 \mathrm{~Hz}\right)$. In contrast, irregular afferents display larger phase leads that increase from $\sim 40^{\circ}$ at $0.5 \mathrm{~Hz}$ to $\sim 70^{\circ}$ at $16 \mathrm{~Hz}$.

\section{Otolith afferent thresholds are independent of resting discharge regularity and stimulation frequency}

We next used signal-detection theory to measure neuronal thresholds based on the activity of single otolith afferents. Based on previous studies (Sadeghi et al., 2007; Massot et al., 2011), we hypothesized that threshold values should strongly depend on both sensitivity and resting discharge regularity. To test this, we determined the detection threshold of each individual afferent by plotting its time-dependent firing rate as a function of linear acceleration (Fig. 3A, top and bottom). Next, we used signal-detection theory to compare the firing rate distribution for a given value of linear acceleration to that obtained for zero acceleration using the $d^{\prime}$ measure (see Materials and Methods; note that this methodology assumes that the firing rate distribution is normal). $d^{\prime}$ was then plotted as a function of linear acceleration (Fig. $3 A$, middle), and the detection threshold, $T_{d^{\prime}}$, was computed as the lowest absolute acceleration value for which $d^{\prime}=1$ (Fig. 3A, blue arrow).

Surprisingly, we found that regular and irregular afferents displayed detection thresholds that were not significantly different from one another $(t$ test with Bonferroni's correction, $p>0.11$ for every frequency of the stimuli). Moreover, threshold values did not significantly vary over the frequency range for both afferent groups $(0.5-16 \mathrm{~Hz})($ ANOVA, $p=0.23$ and $p=0.14$ for regular and irregular afferents, respectively). We also found that detection threshold values obtained when only considering positive or negative linear accelerations, $T_{d^{\prime} \text { exc }}$ and $T_{d^{\prime} \text { inh }}$, respectively, were not significantly different from $T_{d^{\prime}}$ (ANOVA, $p>0.4$ for all stimulus frequencies). This result is expected because the stimuli used were designed to not elicit nonlinear responses from afferents.

To better compare our estimates with (1) values reported previously in the literature (Yu et al., 2012) and (2) perceptual thresholds estimated using different assumptions in behavioral studies (Walsh, 1961, 1962; Young and Meiry, 1968; Jones and Young, 1978; Benson et al., 1986; Zupan and Merfeld, 2008; MacNeilage et al., 2010a,b; Naseri and Grant, 2012; Valko et al., 2012), we also used ROC analysis to estimate both detection (Fig. 4A) and discrimination (Fig. $4 B$ ) thresholds. To compute detection thresholds, we plotted the time-dependent firing rate as a function of linear acceleration (Fig. 4A) and used ROC analysis to compute the probability of correct detection from the firing rate distribution for a given value of linear acceleration $\ddot{H}$ and that obtained for zero acceleration (i.e., $\ddot{H}=0$ ). The probability of correct detection was then plotted as a function of linear acceleration (i.e., the neurometric curve; Fig. 4A, inset). The detection threshold, $T_{\max }$, was defined as the value of linear acceleration corresponding to a probability of correct detection of 0.84 (Fig. 4A, inset, blue arrow). To compute discrimination threshold, we also plotted the time-dependent firing rate as a function of linear acceleration (Fig. $4 B$ ) but instead used ROC analysis to compute the probability of correct detection from the firing rate distributions for a given value of head acceleration $\ddot{H}$ and that obtained for the opposite value $-\ddot{H}$. The corresponding neurometric curve (Fig. $4 B$, inset) was then used to determine the discrimination threshold, $T_{\min -\max }$. Specifically, $T_{\min -\max }$ was defined as the value

\section{A Detection thresholds computation: $d^{\prime}=1$}
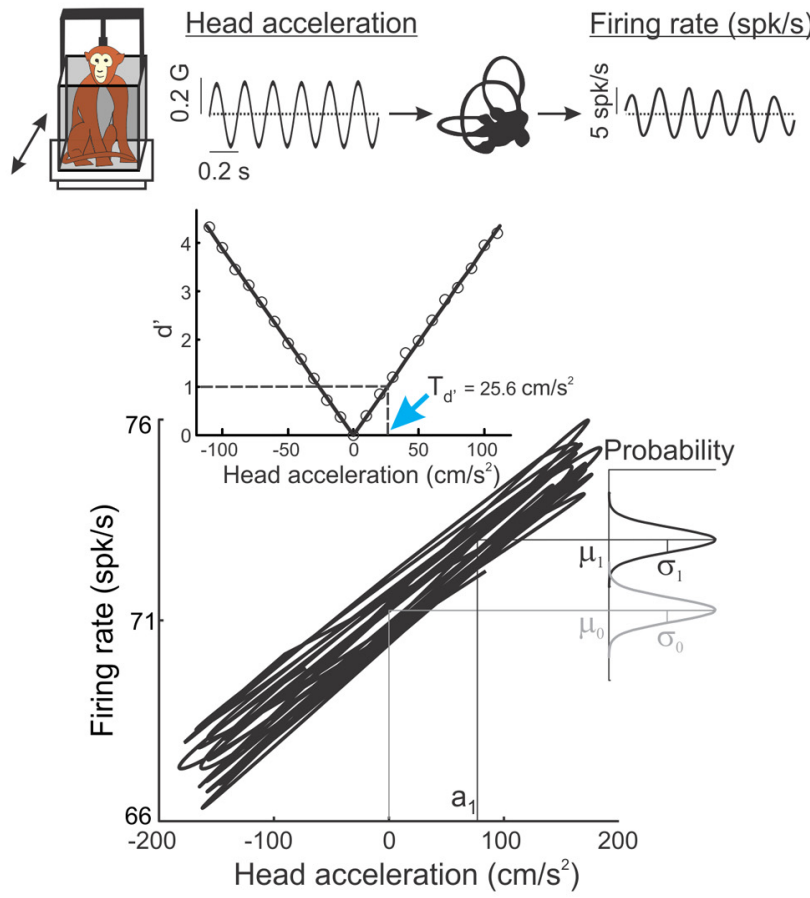

\section{B Average thresholds using $\mathrm{d}^{\prime}=1$}

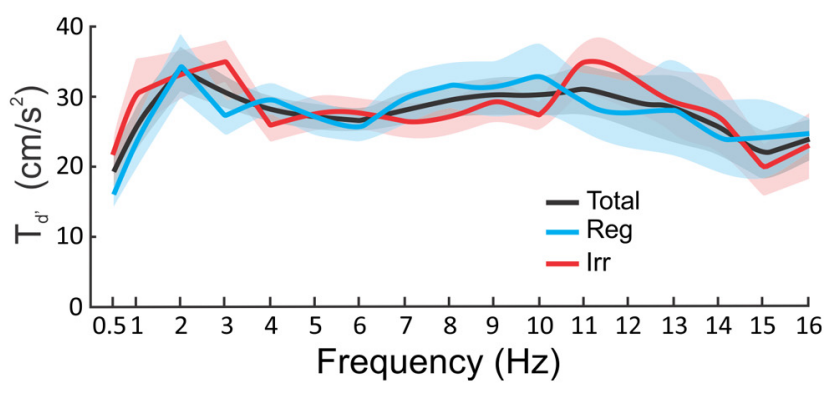

Figure 3. Regular and irregular otolith afferents display similar detection thresholds. $\boldsymbol{A}$, Segment of a $5 \mathrm{~Hz}$ sinusoidal stimulus (top, left) and the corresponding firing rate response (top, right). We plotted firing rate as a function of linear acceleration (bottom) and used signaldetection theory to compare the distribution of firing rates for a given value of linear acceleration (black Gaussian curve) and that obtained for zero acceleration (gray Gaussian curve) to obtain a plot of $d^{\prime}$ as a function of linear acceleration (middle). The detection threshold $T_{d^{\prime}}$ was computed as the lowest absolute value of head acceleration for which $d^{\prime}=1$ (blue arrow). $\boldsymbol{B}$, Population-averaged detection threshold values $T_{d^{\prime}}$ for regular (blue, Reg), irregular (red, Irr), and all (black, Total) afferents as a function of stimulus frequency. It is seen that regular and irregular afferents have similar thresholds ( $t$ test, $p>0.11$ for every stimulus frequency) that do not vary significantly with frequency (ANOVA, $p=0.23$ and $p=0.14$ for regular and irregular afferents, respectively).

of linear acceleration corresponding to a probability of correct detection of 0.84 (Fig. 4B, inset, blue arrow).

We found that detection $T_{\max }$ and discrimination $T_{\min -\max }$ threshold values were independent of stimulus frequency (ANOVA, $p>0.05$ for all conditions), similar to $T_{d^{\prime}}, T_{d^{\prime} \mathrm{exc}}$, and $T_{d^{\prime} \text { inh }}$ (Fig. 4C, top). Importantly, theoretical relationships can be derived between threshold values obtained using different methodologies (see Materials and Methods and Merfeld, 2011). These theoretical calculations predict that threshold estimates will differ by proportionality constants that are independent of stimulus frequency. We found that scaling our threshold estimates by the 
A ROC analysis: $T_{\max }$

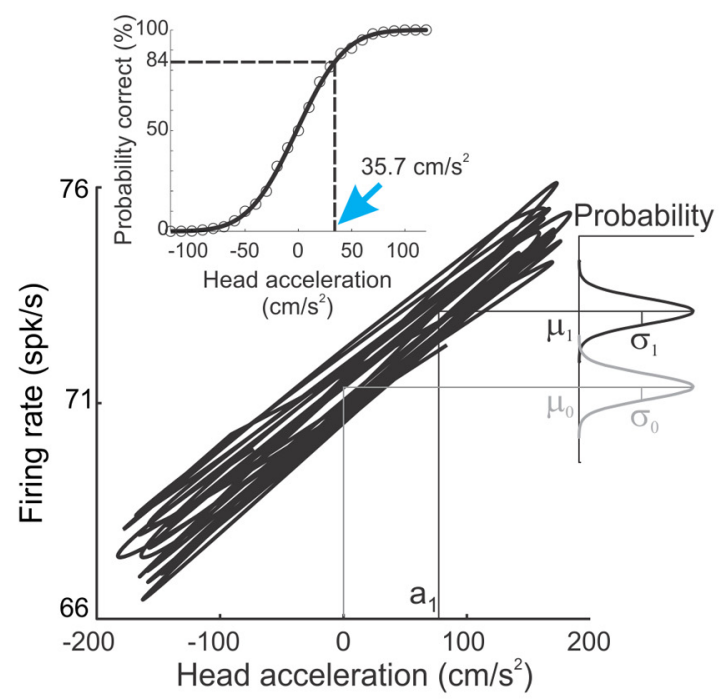

\section{Thresholds: different methods}

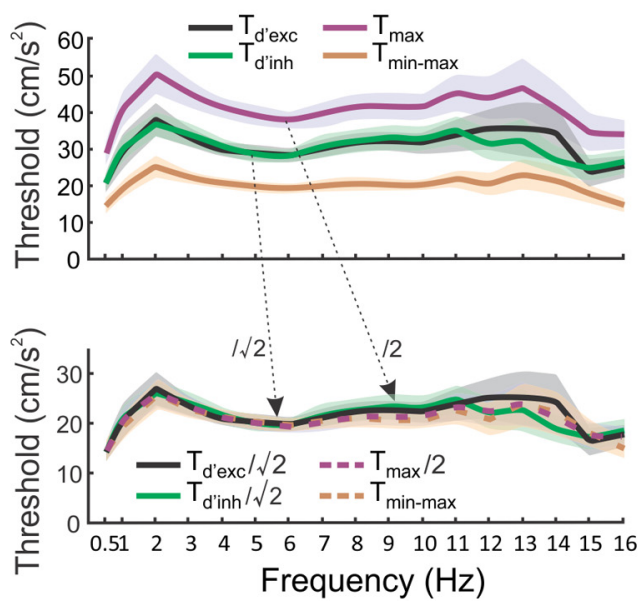

B ROC analysis: $T_{\min -m a x}$

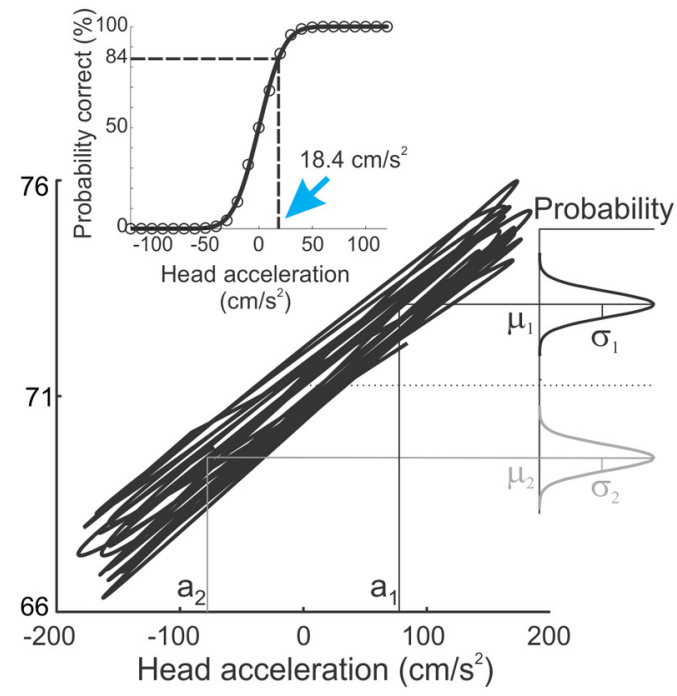

D Thresholds: different decoding

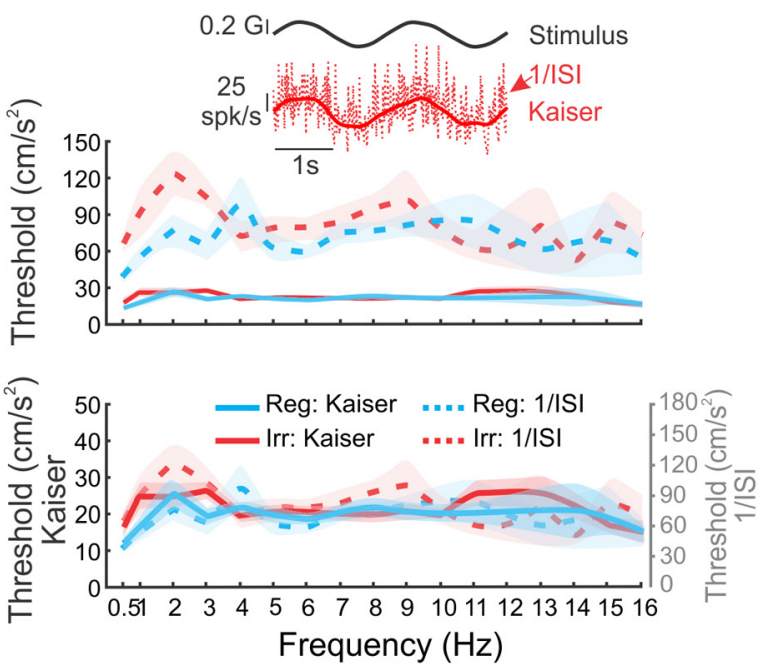

Figure 4. Comparison between neural thresholds obtained using different methodologies for otolith afferents. $A, B$, Plots of the instantaneous firing rate as a function of linear acceleration for the same example regular afferent. We compared the firing rate distributions for a given positive value of acceleration $\ddot{H}$ (black Gaussian curves) and that obtained for zero linear acceleration (i.e., $\ddot{H}=0$ ) (gray curve in $\boldsymbol{A}$ ) or the firing rate distribution when the acceleration has the opposite value (i.e., $-\ddot{H}$ ) (gray curve in $\boldsymbol{B}$ ). The insets illustrate the corresponding neurometric functions obtained using ROC analysis. The detection $T_{\max }$ and discrimination $T_{\min -\max }$ thresholds were computed as the values of linear acceleration for which the probability of correct detection is equal to $84 \%$ (blue arrows in $\boldsymbol{A}$ and $\boldsymbol{B}$, respectively). C, Top, Population-averaged neural threshold values computed using different methodologies: $T_{d^{\prime} \text { exc }}$ (black) and $T_{d^{\prime} \text { inh }}$ (green) were computed using $d^{\prime}$ as described in Figure 3 but using only positive or negative values of head acceleration, respectively. $T_{\max }$ (purple) and $T_{\min -\max }$ (orange) are also shown for comparison. Bottom, Same measures but normalized such that both $T_{d^{\prime} \text { exc }}$ and $T_{d^{\prime} \text { inh }}$ are divided by $\sqrt{2}$ and $T_{\max }$ is divided by 2. It is seen that all curves then coincide (ANOVA, $p>0.54$ for all stimulus frequencies), which illustrates that the different methodologies used to compute neural thresholds lead to estimates that differ only by a proportionality constant that is independent of frequency. $\boldsymbol{D}$, Top, Population-averaged $T_{\min -\max }$ values for regular (blue, Reg) and irregular (red, Irr) afferents obtained when the firing rate is estimated using the inverse ISI (dashed) and a Kaiser filter (solid). It is seen that using a Kaiser filter gives rise to significantly lower threshold estimates ( $t$ test, $p<0.05$ for all frequencies). However, both regular and irregular afferents display thresholds that are not significantly different from one another using either methodology ( $t$ test, Kaiser filter, $p>0.05$ for all frequencies; $1 / / \mathrm{SI}, p>0.05$ for all frequencies). The inset illustrates the firing rates estimated by the reciprocal ISI (dashed red) and Kaiser filter (solid red) to a $0.5 \mathrm{~Hz}$ sinusoidal head acceleration stimulus (solid black). Bottom, Same quantities as a function of stimulus frequency using a double $y$-axis plot. The fact that all curves superimpose illustrates that using different methodologies to estimate firing rate will give rise to threshold estimates that only differ by a proportionality constant that is independent of frequency. We found empirically that dividing the threshold values estimated from the inverse ISI by 3.6 gave rise to values that were not significantly different from those obtained using the Kaiser filter ( $t$ test, $p>0.13$ and $p>0.07$ for regular and irregular afferents, respectively, for every stimulus frequency). The shaded bands represent 1 SEM.

appropriate constants make them coincide for all stimulus frequencies (Fig. $4 C$, bottom; ANOVA, $p>0.54$ for every frequency of the stimuli tested). Importantly, all threshold estimates were not significantly different between regular and irregular afferents regardless of the methodology used ( $t$ test with Bonferroni's correction, $p>0.05$ for every frequency of the stimuli). Thus, we will only report discrimination threshold values $T_{\min -\max }$ below.

\section{Discrimination threshold values depend on firing rate estimation}

We next explored whether our threshold estimates depend on the specific computation used to estimate the time-dependent firing rate. Previous studies have shown that filtering the spike train to estimate the firing rate will significantly influence threshold estimates (Jamali et al., 2009; Yu et al., 2012). We compared 
threshold estimates obtained using two different methodologies that are at the opposite ends of the spectrum: (1) extensive filtering using a Kaiser window (Jamali et al., 2009; Yu et al., 2012) and (2) no filtering by using reciprocal ISI (Jamali et al., 2009; Yu et al., 2012). Both methodologies are illustrated in the inset of Figure $4 D$. This comparison is important because previous studies have shown that threshold estimates obtained using other filters were higher than those obtained using a Kaiser filter (Jamali et al., 2009; Yu et al., 2012). Thus, the Kaiser filter allows us to obtain a lower-bound estimate of neural threshold. Threshold values computed using the inverse ISI were much larger than those computed using a Kaiser filter (Fig. 4D, top). However, threshold values obtained using the inverse ISI or Kaiser filter were not significantly different when compared between regular and irregular afferents ( $t$ test with Bonferroni's correction, $p>0.05$ for every stimulus frequency). Moreover, our results show that threshold values obtained using both approaches differed only by an empirical proportionality constant $(\sim 3.6)$ that is independent of frequency (Fig. $4 D$, bottom). Indeed, normalizing by this constant made both threshold estimates coincide for all frequencies ( $t$ test with Bonferroni's correction, $p>0.13$ and $p>0.07$ for regular and irregular afferents, respectively).

\section{Comparison with neural threshold values reported previously in the literature}

Our measured threshold values are substantially higher than those reported previously by Yu et al. (2012). However, it is important to note that we directly measured thresholds by stimulating each afferent along its PD, whereas Yu et al. (2012) linearly extrapolated threshold values in the PD from values that they obtained from stimulation along the fore-aft and lateral axes. To make a direct comparison between the two studies, we extrapolated discrimination threshold values in the PD from values measured during stimulation along the fore-aft and lateral axes. Previous studies have shown that the response sensitivity of otolith afferents as a function of direction is well fit by a cosine function (Fig. 5A, inset) (Fernández and Goldberg, 1976a; Angelaki and Dickman, 2000). Thus, given that discrimination threshold is inversely related to sensitivity (see Materials and Methods), we would then expect that a plot of threshold as a function of direction would be well fit by an inverse cosine function (Fig. 5A).

Accordingly, we plotted the measured threshold values as a function of the absolute difference between the axis of translation (i.e., fore-aft or lateral) and PD (Fig. 5B, compare with Yu et al., 2012, their Fig. $4 D$ ). We found that our data were well fit by an inverse cosine function $\left(R^{2}=0.9\right.$ and 0.5 for irregular and regular afferents, respectively). We then extrapolated discrimination threshold values in the PD and found average threshold values of 16.3 and $17.1 \mathrm{~cm} / \mathrm{s}^{2}$ for regular and irregular afferents, respectively. We note that these values were not significantly different from those directly obtained from stimulation along PD ( $t$ test, $p=0.14$ and 0.09 for regular and irregular afferents, respectively; Fig. $5 B$, inset). We then fit our data using a linear function, as done by Yu et al. (2012) (Fig. 5C). We found that fitting a linear function gave rise to lower $R^{2}$ values $\left(R^{2}=0.22\right.$ and 0.21 for irregular and regular afferents, respectively) than fitting an inverse cosine function. Furthermore, the extrapolated threshold values in the PD were then 9.8 and $10.5 \mathrm{~cm} / \mathrm{s}^{2}$ for regular and irregular neurons, respectively (Fig. $5 C$ ). These were significantly lower than values directly obtained from stimulation along $\mathrm{PD}(t$ test, $p<10^{-3}$ for both regular and irregular afferents; Fig. $5 C$, inset). Thus, this analysis suggests that Yu et al. (2012) signifi- cantly underestimated neural discrimination threshold values along PD because of a linear extrapolation procedure.

Finally, we note that Yu et al. (2012) reported threshold estimates in the excitatory and inhibitory directions $\left(T_{d^{\prime} \text { exc }}\right.$ and $T_{d^{\prime} \text { inh }}$, respectively) that were quite different from one another for some afferents in their dataset. Yu et al. (2012) attributed these differences to nonlinearities in the response (see their Fig. 7). In contrast, we did not observe such nonlinearities in our dataset. This could be attributable to the fact that we directly measured discrimination thresholds in the PD of each afferent, which implies that each afferent was well driven by the stimulus. Nevertheless, we note that, when Yu et al. (2012) extrapolated threshold values along PD, they found no significant differences between threshold estimates for the excitatory and inhibitory directions, in agreement with our results.

\section{Strong covariation between sensitivity and variability leads to discrimination thresholds that are independent of resting discharge regularity and stimulus frequency}

We next investigated the reasons why regular and irregular otolith afferents, despite having large heterogeneities in resting discharge regularity, displayed similar detection/discrimination thresholds. Theoretical calculations show that, if the firing rate distribution for a given linear acceleration $\ddot{H}$ is a Gaussian whose variance $\sigma^{2}$ is independent of $\ddot{H}$, which is verified by our dataset (Fig. 3A, bottom), then the discrimination threshold is proportional to the ratio of the $\mathrm{SD}$ (i.e., $\sigma$ ) divided by the sensitivity (i.e., the slope of the firing rate as a function of linear acceleration curve; Eq. 4; see Materials and Methods).

To test this prediction, we superimposed plots of response sensitivity and trial-to-trial variability as quantified by the SD of the firing rate distribution at zero acceleration as a function of resting discharge regularity as quantified by $\mathrm{CV}^{\star}$ (Fig. $6 A$ ). We found a strong positive linear correlation between variability and sensitivity for our dataset (Fig. $6 B ; r=0.9, p \ll 10^{-3}$, slope $=$ $0.024 \pm 0.009 \mathrm{G}$, offset $=0.5 \pm 2.3$ spikes/s). Importantly, the offset was not significantly different from $0(p=0.4)$, indicating that sensitivity is not merely linearly correlated with but is actually proportional to variability. We note that variability and sensitivity were also significantly positively correlated when both regular $\left(r=0.75, p \ll 10^{-3}\right)$ and irregular $(r=0.45, p=0.04)$ afferents were considered separately and that the slope and offset values obtained for each group were not significantly different from those obtained for the full dataset $(p>0.14, t$ tests with Bonferroni's correction).

The fact that variability is proportional to sensitivity indicates that their ratio should be constant. We thus computed predicted discrimination threshold values, $T_{\text {pred }}$, which are proportional to the ratio of variability to sensitivity (see Materials and Methods), and compared them with the actual measured discrimination threshold values $T_{\min -\max }$ as a function of $\mathrm{CV}^{\star}$ (Fig. $6 \mathrm{C}$ ). We found excellent agreement between both quantities, as confirmed by plotting $T_{\text {pred }}$ as a function of $T_{\min -\max }$, which revealed that all data points were close to the identity line (Fig. $6 \mathrm{C}$, inset). Both measures were not significantly different from one another because the slope $(0.96 \pm 0.15)$ and offset $(2.3 \pm 3.7)$ of the linear regression were not significantly different from $1(p=0.3)$ and 0 $(p=0.4)$, respectively. We also measured variability using the resting discharge and found a strong positive correlation with sensitivity (Fig. $6 D ; r=0.8, p \ll 10^{-3}$ ), and the offset of the linear fit $(0.99 \pm 3.18$ spikes/s $)$ was not significantly different from 0 ( $p=0.4$ ), further supporting our hypothesis that variability is proportional to sensitivity across otolith afferents. 


\section{A Hypothetical neuron}
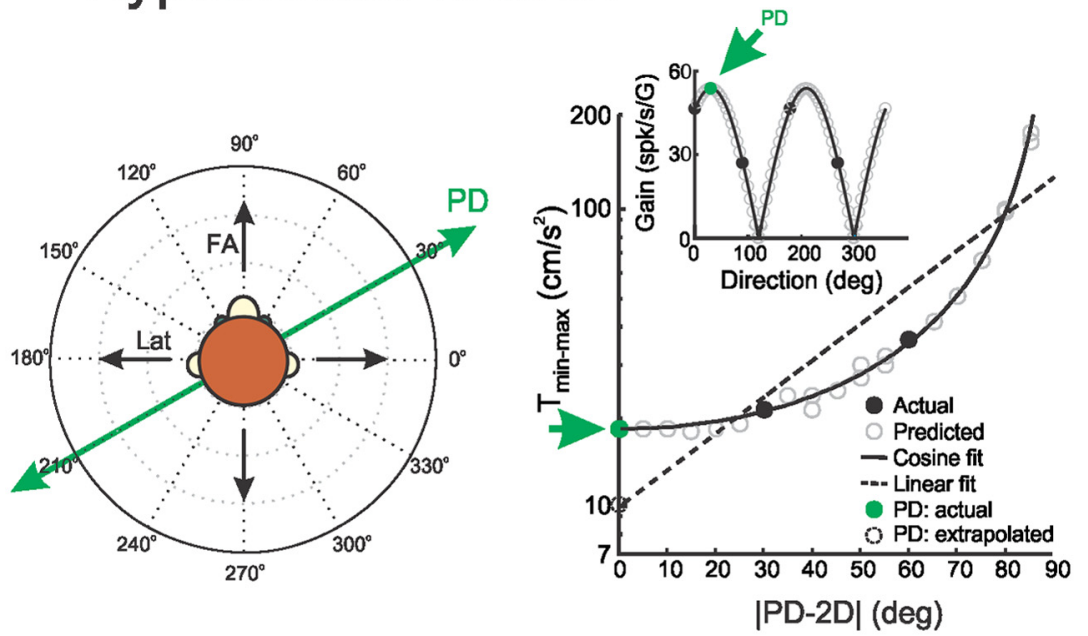

B 1/Cosine fit

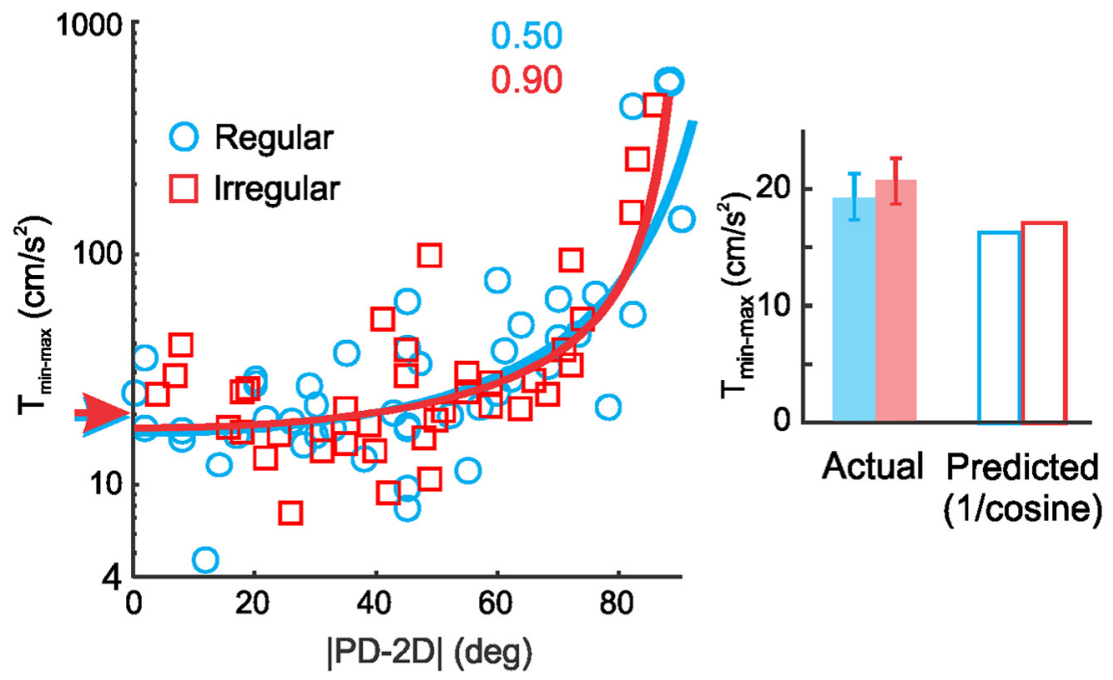

\section{Linear fit}
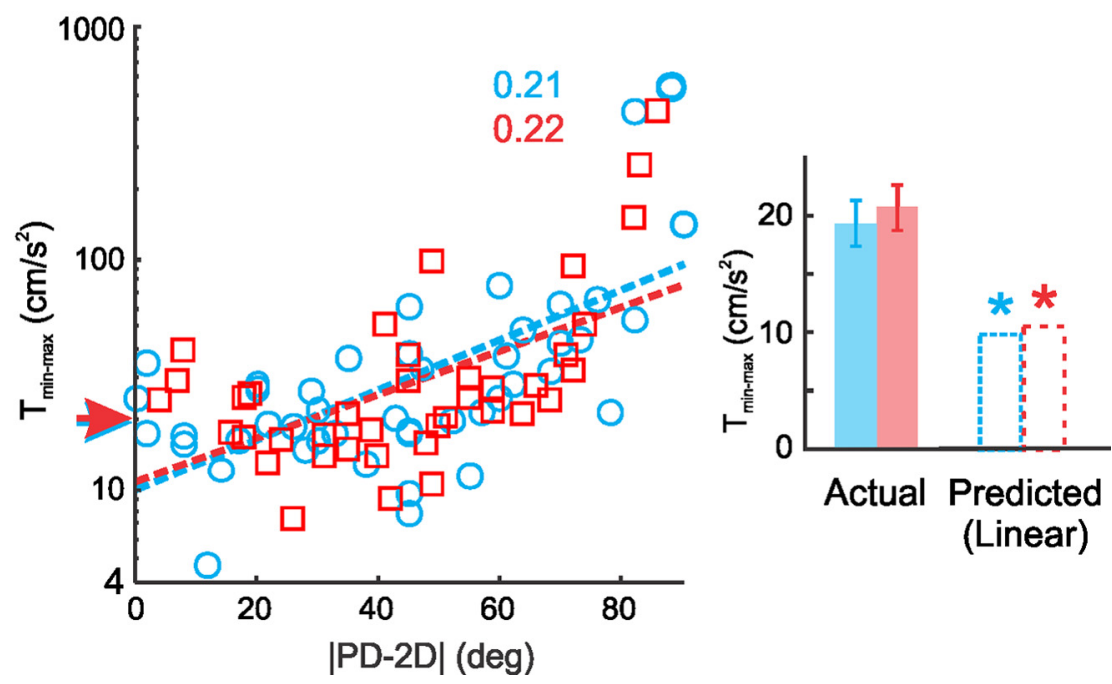

Figure 5. Linear extrapolation significantly underestimates neural threshold values in the PD. $\boldsymbol{A}$, Illustration showing why linear extrapolation would underestimate the neural discrimination threshold at PD. Left, Schematic of the fore-aft (FA) and
We next tested whether proportionality between variability and sensitivity could also explain our results showing that discrimination thresholds are independent of stimulus frequency. To do so, we superimposed plots of sensitivity and variability as a function of stimulus frequency (Fig. 6E). Our results show that both quantities are proportionally related: there is a strong positive linear correlation for regular $\left(r=0.82, p<10^{-3}\right)$ and irregular $\left(r=0.85, p<10^{-3}\right)$ afferents, with the offsets not significantly different from $0(p>0.4)$. This suggests that the ratio of variability to sensitivity is also constant across stimulus frequencies. We therefore used the measured values of variability and sensitivity to predict the discrimination threshold as done above and compared predicted values, $T_{\text {pred }}$, with actual measured values, $T_{\min -\max }$, as a function of stimulus frequency. We found that both measures were not significantly different from one another for both irregular (Fig. 6F, top) and regular (Fig. 6F, bot-

lateral (Lat) axes of translation (black arrows) as well as the PD of an example afferent (green line and arrows). Right, Discrimination threshold $\left(T_{\min -\max }\right)$ of this afferent as a function of direction (i.e., the absolute difference between the axis of translation and the PD of the afferents: $|P D-2 D|$ ) calculated using the sensitivity tuning curve (inset). The black circles show measured sensitivities (inset) and corresponding threshold values (main panel). The gray circles were obtained using a cosine fit (solid black line in inset) for sensitivity (inset) and estimating the corresponding threshold values. The predicted threshold at PD (dashed circle) based on a linear extrapolation (dashed line) is much smaller than the actual value measured by direct stimulation along the PD of the afferent (filled green circle, green arrow). Based on the fact that the discrimination threshold is inversely proportional to sensitivity, we would expect that the threshold as a function of direction would be well fit by a $1 /$ cosine function (solid black line in main panel). Extrapolating the discrimination threshold at PD using this fit gives rise to a correct estimate. $\boldsymbol{B}$, Discrimination threshold values $T_{\min -\max }$ as a function of direction for regular (blue) and irregular (red) afferents during $5 \mathrm{~Hz}$ sinusoidal stimulation. We used a $1 /$ cosine fit (solid lines; see Materials and Methods) to extrapolate the threshold at PD (red and blue arrows). The $R^{2}$ values quantifying the goodness-of-fit are also shown. We then compared the extrapolated threshold values (open bars in the inset) with the actual measured values (filled bars in the inset) and found no significant differences ( $t$ test, $p=0.14$ and 0.09 for regular and irregular afferents, respectively). $\boldsymbol{C}$, Same data as in $\boldsymbol{B}$ except that we instead used a linear fit to extrapolate discrimination threshold values at PD. The best fits are shown (dashed lines) along with goodnessof-fit as quantified by $R^{2}$. We note that fitting a straight line gave rise to lower $R^{2}$ values than using a $1 /$ cosine function. We also compared the linearly extrapolated discrimination threshold values (red and blue arrows, open bars in the inset) with the actual measured values (filled bars in the inset) and found that the former significantly underestimated the latter ( $t$ test, $p<0.0001$ for both regular and irregular afferents). 
tom) afferents ( $t$ test with Bonferroni's correction, $p>0.14$ for all frequencies).

In conclusion, our results show that, because variability is proportional to sensitivity, increases in the former are effectively compensated by increases in the latter when either (1) varying stimulus frequency for a given afferent or (2) comparing afferents with different resting discharge regularities, such that their ratio remains constant. This leads to discrimination thresholds that are independent of either resting discharge regularity or stimulus frequency across the otolith afferent population.

Pooling the activities of otolith afferent populations of increasing size decreases discrimination threshold

Our results show that individual otolith afferents display discrimination thresholds that are substantially higher (at least 12 times) than values reported in perceptual studies (Benson et al., 1986; Zupan and Merfeld, 2008; MacNeilage et al., 2010a,b; Naseri and Grant, 2012; Valko et al., 2012). Accordingly, this implies that perceptual thresholds are achieved by integrating the activities of multiple afferents by higherorder brain areas. We used Fisher information to estimate the population size from which information must be integrated to give rise to perception. Because our results show that regular and irregular afferents have similar discrimination thresholds, the data from both groups were pooled for this analysis.

We found that neural discrimination threshold values decreased as a function of increasing population size $(N)$ both when the firing rate was estimated via a Kaiser filter (Fig. 7A) and via the inverse ISI (Fig. 7B). Furthermore, we found that the curves relating the discrimination threshold to population size were well fitted by a $1 / \sqrt{N}$ function in both cases $\left(R^{2}=0.93\right.$ and 0.9 for Fig. 7, $A$ and $B$, respectively). This is expected (Seung and Sompolinsky, 1993) given that there is no correlation between the variabilities of different otolith afferents as discussed below. Using this fit, we extrapolated the population size for which the neural discrimination threshold value is equal to perceptual values reported by MacNeilage et al. (2010b) and Valko et al. (2012). We only compared our neural threshold values to perceptual threshold values from these studies because they used experimental paradigms that were most similar to our own as discussed below.

When we used a Kaiser filter to estimate the firing rate, we found that population sizes of 7 and $\sim 120$ afferents led to
A

$\underline{5 \mathrm{~Hz} \text { translation }}$

B

$\underline{5 \mathrm{~Hz} \text { translation }}$

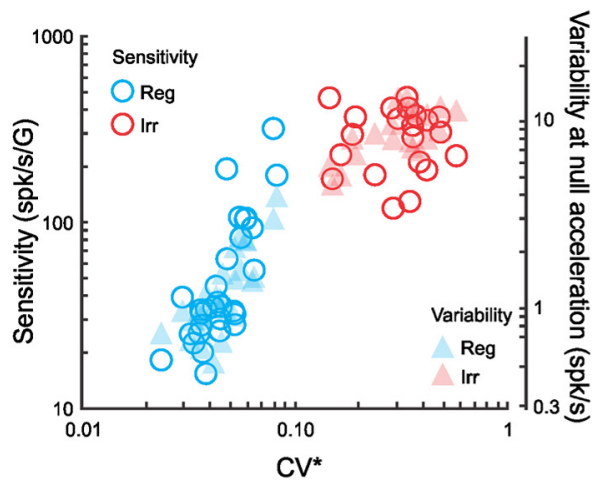

C

$5 \mathrm{~Hz}$ translation

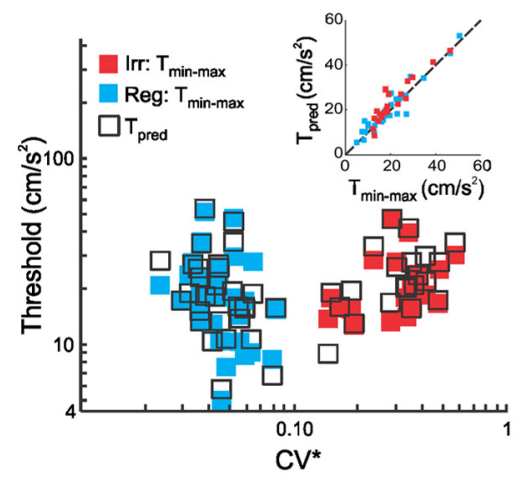

$\mathrm{E}$

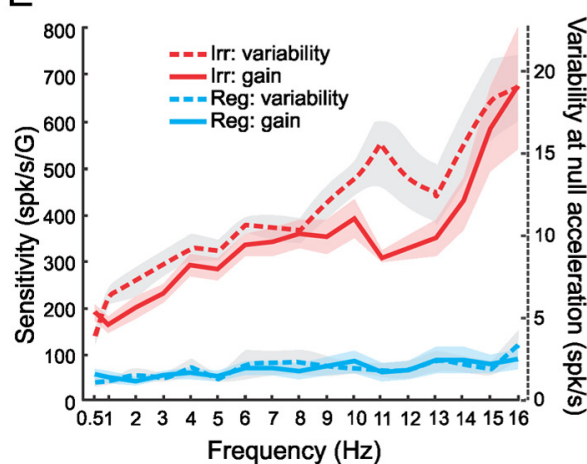

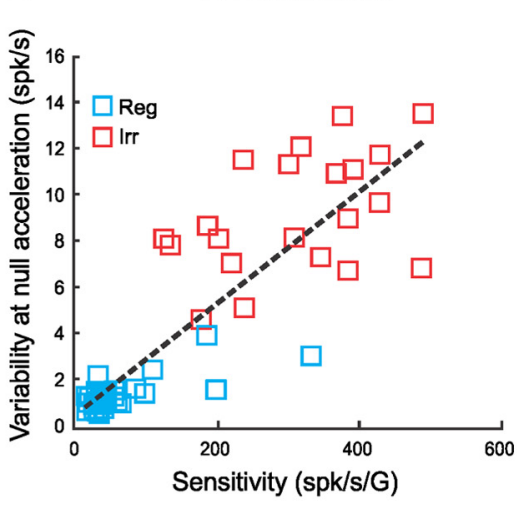

D $\underline{5 \mathrm{~Hz} \text { translation }}$

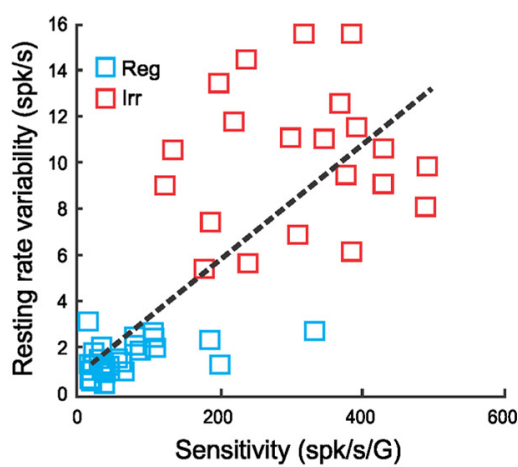

F

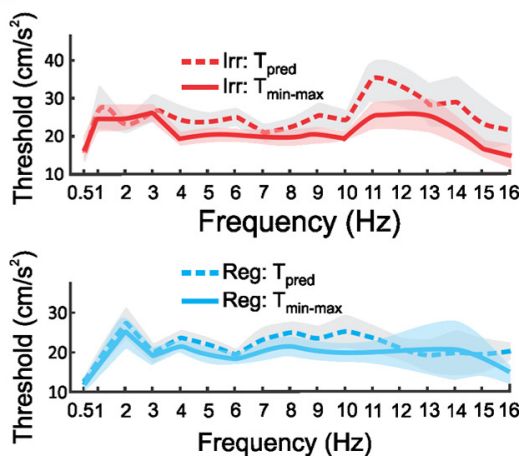

Figure 6. Strong positive correlations between sensitivity and variability lead to discrimination thresholds that are independent of resting discharge regularity and stimulus frequency. $A$, Response sensitivity as quantified by the slope of the firing rate-head acceleration curve (circles) and variability as quantified by the SD of the firing rate distribution obtained via a Kaiser filter at zero head acceleration (triangles) as a function of $\mathrm{CV}^{*}$ for regular (blue, Reg) and irregular (red, Irr) afferents during $5 \mathrm{~Hz}$ sinusoidal stimulation. $\boldsymbol{B}$, Variability as a function of sensitivity for regular (blue) and irregular (red) afferents during $5 \mathrm{~Hz}$ sinusoidal stimulation. Both quantities were strongly positively correlated $(r=0.9, p$ $\left.\ll 10^{-3}\right)$. The linear fit to the data (dashed line) had a slope of $0.024 \pm 0.009 \mathrm{G}$ and an offset of $0.5 \pm 2.3$ spikes $/ \mathrm{s}$. Importantly, the offset was not significantly different from $0(p=0.4)$, indicating that variability is proportional to sensitivity. C, Comparison between discrimination threshold values $T_{\min -\max }$ for regular (filled blue rectangles) and irregular (filled red rectangles) afferents as a function of $\mathrm{CV}^{*}$. Also shown are the predicted threshold values based on sensitivity and variability (open black rectangles). The inset shows the predicted discrimination threshold values as a function of the actual measured ones. There is excellent agreement because all data points are scattered close to the identity line (dashed line) $\left(R^{2}=0.89\right.$, the slope of the best-fit straight line is not significantly different from $\left.1, p=0.44\right)$. $D$, A strong positive correlation between resting rate variability and neuronal sensitivity $\left(r=0.8, p \ll 10^{-3}\right)$ : the slope of the best linear regression was $0.025 \pm 0.013 \mathrm{G}$ and the offset $(0.99 \pm 3.18$ spikes $/ \mathrm{s})$ was not significantly different from $0(p=0.4)$. Note that resting rate variability was estimated as the SD of the Kaiser filtered firing rate in the absence of any stimulus. E, Population-averaged response sensitivity (solid) and variability (dashed) as a function of linear acceleration frequency for regular (blue) and irregular (red) afferents. $\boldsymbol{F}$, Population-averaged discrimination threshold values $T_{\min -\max }$ (solid) did not significantly differ from the predicted values (dashed) over the whole frequency range for both regular (bottom) ( $t$ test, $p>0.31$ for all stimulus frequencies) and irregular (top) ( $t$ test, $p>0.15$ for all stimulus frequencies) afferents. The shaded bands represent 1 SEM. 


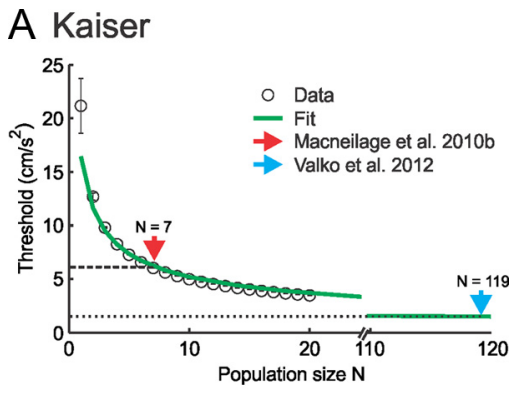

B $1 /|S|$

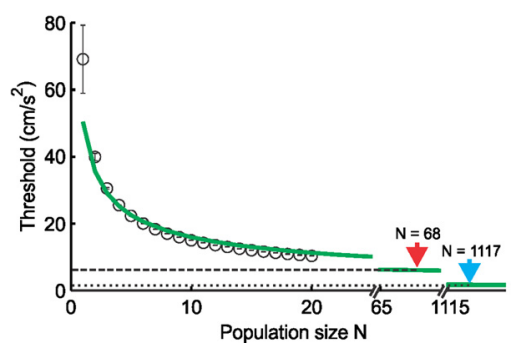

Figure 7. Pooling the activities of afferent populations of increasing size gives rise to decreasing discrimination threshold values that approach those reported in perceptual studies. $A$, $B$, Population-averaged discrimination threshold values (circles) $T_{\min -\max }$ as a function of population size $N$ obtained by estimating the firing rate using either a Kaiser filter $(\boldsymbol{A})$ or the inverse ISI (B). Also shown are a $1 / \sqrt{ } N$ fit (green traces in $\boldsymbol{A}$ and $\boldsymbol{B}$ ) as well as perceptual threshold values reported by MacNeilage et al. (2010b) (dashed line) and Valko et al. (2012) (dotted line). In both cases, the data are well fit $\left(\boldsymbol{A}, R^{2}=0.93 ; \boldsymbol{B}, R^{2}=0.90\right)$, indicating that the discrimination threshold decreases as $1 / \sqrt{ } N$. In both panels, the red and blue arrows give the smallest population sizes that lead to discrimination threshold values that are equal to those reported for perception by MacNeilage et al. (2010b) and Valko et al. (2012), respectively.

threshold values equal to those reported by MacNeilage et al. (2010b) and Valko et al. (2012), respectively. In contrast, when the firing rate was estimated using the inverse ISI, the population sizes were significantly larger $(\sim 70$ and $\sim 1100$, respectively). Thus, our results show that perceptual thresholds for linear acceleration are most likely achieved by integration of the activities of large afferent populations by higher-order areas.

\section{Discussion}

In the present study, we addressed the important question of how information transmitted by the vestibular periphery determines the ability to discriminate linear motion. Specifically, we focused on whether higher-order areas must integrate the activities of otolith afferent populations to give rise to perception. We found that threshold values obtained when linear motion was applied along the PD of each afferent were constant as a function of frequency $(0.5-16 \mathrm{~Hz})$ as well as resting discharge regularity. This was because increased trial-to-trial variability was effectively matched by increased sensitivity. Our threshold estimates from single afferents were approximately $\sim 20$ and $\sim 76 \mathrm{~cm} / \mathrm{s}^{2}$ when the firing rate was estimated via a Kaiser filter and 1/ISI, respectively. These values were at least an order of magnitude greater than perceptual thresholds of $\sim 1.5 \mathrm{~cm} / \mathrm{s}^{2}$ as reported by Valko et al. (2012). Thus, our results suggest that higher-order vestibular pathways must integrate the activities of large afferent populations to give rise to perception.

\section{Estimation of neuronal thresholds}

Our results show that different methodologies lead to different neuronal threshold estimates. In particular, because of extra filtering that removes variability from the firing rate estimate, using a Kaiser window resulted in values that were consistently $\sim 3.6$ times lower than those obtained using the reciprocal ISI. Thus, to evaluate thresholds for single afferents, the reciprocal ISI methodology is most likely more appropriate. However, filtering via a Kaiser window might approximate the mechanisms by which afferent spike trains are actually integrated by downstream neurons. Additional studies directly measuring thresholds from neurons within the vestibular nuclei receiving input from otolith afferents are necessary to verify this hypothesis. We also note that, although we used the Kaiser filter to obtain a lower-bound estimate of neural thresholds, other forms of filtering (e.g., a Gaussian window) have been explored previously and give rise to greater values than those reported using a Kaiser filter (Yu et al., 2012). However, in contrast to the present study, Yu et al. (2012) estimated threshold values in the PD of each afferent using a linear extrapolation rather than by direct measurement (Yu et al., 2012, their Figs. 4D and 6D). Our current analysis demonstrates that the approach by Yu et al. (2012) significantly underestimates neural threshold values (Fig. 5) because it does not take into account the cosine dependency of the tuning curves of individual otolith afferents (Fernández and Goldberg, 1976a; Angelaki and Dickman, 2000).

\section{Comparison between neural and perceptual thresholds}

To compare our neuronal threshold values with those measured in psychophysical studies of linear self-motion, we focused on the most recent studies that used stimulation paradigms that are most similar to our own (MacNeilage et al., 2010b, Valko et al., 2012). We note that our neural threshold values were obtained in Macaque monkeys, whereas perceptual values were obtained in humans. Such comparisons must be made cautiously, in general, because previous studies have found quantitative differences between vestibular responses in humans and monkeys (Lansberg et al., 1964; Merfeld and Young, 1995; Merfeld et al., 2001). However, more recent studies suggest that the mechanisms that lead to perception are comparable in both species (Merfeld and Zupan, 2002). Thus, it is of interest to compare neural threshold values obtained in monkeys to perceptual threshold values obtained in humans as has been done in recent studies (Haburcakova et al., 2012; Yu et al., 2012).

On average, we found that our lower-bound discrimination threshold values for single afferents $\left(19.2 \pm 2.2 \mathrm{~cm} / \mathrm{s}^{2}\right.$ at $\left.1 \mathrm{~Hz}\right)$ were at least three times larger than perceptual values measured in either behavioral study (MacNeilage et al., 2010b, $6.1 \mathrm{~cm} / \mathrm{s}^{2}$; Valko et al., 2012, $1.5 \mathrm{~cm} / \mathrm{s}^{2}$ ). Thus, our results strongly suggest that linear motion perception arises by integration of peripheral otolith afferent population activity by higher-order vestibular areas, which is similar to what has been reported for semicircular canal afferents (Sadeghi et al., 2007; Massot et al., 2011) and other senses (visual: Pelli, 1985; Amano et al., 2006; Shadlen et al., 1996; auditory: Pfingst and Xu, 2004; Bizley et al., 2010; cross modal: McDonald et al., 2000; Shams et al., 2000, 2002). Indeed, our calculation using Fisher information shows that the population size is $\sim 100$ if we use a Kaiser filter to estimate firing rate and the threshold estimates of Valko et al. (2012). It is important to note that our population size estimate is based on a computation that assumes that afferents do not display correlations in their variabilities (i.e., noise correlations) and thus constitutes a lower bound (Averbeck et al., 2006). Indeed, afferent fibers may share common vestibular hair cell input, thereby leading to positively correlated variabilities (Eatock and Songer, 2011), which in turn would increase the population size. 


\section{Impact of variability on neural coding}

The role of trial-to-trial variability in neural coding remains an important problem in systems neuroscience (Stein et al., 2005; McDonnell and Ward, 2011). The peripheral vestibular system is particularly well suited for studying this role because afferent resting discharge regularity varies over a wide range. Our results demonstrate that, although irregular otolith afferents have substantially larger sensitivity than regular ones, both classes display similar thresholds. This is because the increased sensitivity seen for irregular afferents is effectively matched by an increase in their trial-to-trial variability such that the ratio remains constant.

Importantly, we note that a strong positive correlation between sensitivity and variability is not sufficient to result in detection/discrimination thresholds that are independent of resting discharge variability. Indeed, a markedly different coding strategy is used by semicircular canal vestibular afferents, which are instead sensitive to rotations. Although a strong positive correlation between variability and sensitivity is also observed for semicircular canal afferents (Goldberg, 2000), the increased sensitivity displayed by irregular semicircular canal afferents is not sufficient to compensate for their substantially higher trial-to-trial variability. As a result, irregular semicircular canal afferents display higher detection thresholds than regular ones (Sadeghi et al., 2007; Massot et al., 2011).

Why is it advantageous for the otolith system to have two classes of afferents (i.e., regular and irregular) with different sensitivities when these are effectively compensated by differences in variability? As noted above, this result was unexpected because qualitatively different findings were obtained previously for semicircular canal afferents. However, we note that all our analyses consider only steady-state responses and not responses to more transient stimuli. Moreover, our stimuli were designed to not elicit static nonlinearities, such as cutoff and/or saturation. For instance, the predicted stimulus intensity that is required to elicit nonlinear responses from irregular otolith afferents is much smaller than for regular afferents because of their larger sensitivity and the fact that both groups have similar resting discharge rates. Thus, it is likely that transient perturbations will preferentially elicit nonlinear responses from irregular otolith afferents. Additional studies focusing on the strategies used by the otolith system to encode more transient stimuli, in particular with an emphasis on stimuli that produce nonlinear responses, might yield new insight into functional implications of the differences between the two otolith afferent classes.

Our results add to the growing body of literature showing that trial-to-trial variability should be taken into account when studying the neural code (Chacron et al., 2005; Sadeghi et al., 2007; Faisal et al., 2008). The trial-to-trial variability in the response of vestibular afferents to repeated presentations of the same stimulus most likely originates from sensory transduction as well as intrinsic properties (Smith and Goldberg, 1986; Kalluri et al., 2010) that correspond to the "sensory noise" and "cellular noise" categories as described by Faisal et al. (2008). We note that environmental noise (i.e., noise in the stimulus) most likely does not contribute to the measured trial-to-trial variability because we directly measured the head acceleration. In contrast, sensory noise such as thermal or physiological motion (e.g., mechanical gating at the level of channels) at the level of the receptor cells could contribute to the observed trial-to-trial variability in afferents. Furthermore, modeling in this system suggests that this variability is caused, at least in part, by postsynaptic mechanisms (Smith and Goldberg, 1986). It is theoretically possible that there is a source of sensory noise that is common to all otolith afferents and that is greater for irregular afferents because of their larger gains. However, additional studies are needed to test this interesting hypothesis.

Our present results suggest that, in the otolith system, increases in sensitivity are effectively compensated by increases in trial-to-trial variability such that their ratio remains constant, which is qualitatively different from what is observed for semicircular canal afferents. Nevertheless, such a strategy might be found in other sensory processing because correlations between sensitivity and variability are also found in other systems (Kiang, 1965; Tollin et al., 2008).

\section{Conclusion}

We conclude that strong correlations between sensitivity and trial-to-trial variability lead to neuronal thresholds that are independent of frequency and resting discharge regularity in otolith afferents. Furthermore, our results strongly suggest that integration of neuronal activities across a large population of afferents by higher-order vestibular areas is required to give rise to perception.

\section{References}

Amano K, Goda N, Nishida S, Ejima Y, Takeda T, Ohtani Y (2006) Estimation of the timing of human visual perception from magnetoencephalography. J Neurosci 26:3981-3991. CrossRef Medline

Angelaki DE, Dickman JD (2000) Spatiotemporal processing of linear acceleration: primary afferent and central vestibular neuron responses. J Neurophysiol 84:2113-2132. Medline

Averbeck BB, Latham PE, Pouget A (2006) Neural correlations, population coding and computation. Nat Rev Neurosci 7:358-366. CrossRef Medline

Benson AJ, Spencer MB, Stott JR (1986) Thresholds for the detection of the direction of whole-body, linear movement in the horizontal plane. Aviat Space Environ Med 57:1088-1096. Medline

Bizley JK, Walker KM, King AJ, Schnupp JW (2010) Neural ensemble codes for stimulus periodicity in auditory cortex. J Neurosci 30:5078-5091. CrossRef Medline

Chacron MJ, Maler L, Bastian J (2005) Electroreceptor neuron dynamics shape information transmission. Nat Neurosci 8:673-678. CrossRef Medline

Cherif S, Cullen KE, Galiana HL (2008) An improved method for the estimation of firing rate dynamics using an optimal digital filter. J Neurosci Methods 173:165-181. CrossRef Medline

Cullen KE, Minor LB (2002) Semicircular canal afferents similarly encode active and passive head-on-body rotations: implications for the role of vestibular efference. J Neurosci 22:RC226. Medline

Cullen KE, Rey CG, Guitton D, Galiana HL (1996) The use of system identification techniques in the analysis of oculomotor burst neuron spike train dynamics. J Comput Neurosci 3:347-368. CrossRef Medline

Dale A, Cullen KE (2013) The nucleus prepositus predominantly outputs eye movement-related information during passive and active selfmotion. J Neurophysiol 109:1900-1911. CrossRef Medline

Eatock RA, Songer JE (2011) Vestibular hair cells and afferents: two channels for head motion signals. Annu Rev Neurosci 34:501-534. CrossRef Medline

Faisal AA, Selen LP, Wolpert DM (2008) Noise in the nervous system. Nat Rev Neurosci 9:292-303. CrossRef Medline

Fernández C, Goldberg JM (1976a) Physiology of peripheral neurons innervating otolith organs of the squirrel monkey. II. Directional selectivity and force-response relations. J Neurophysiol 39:985-995. Medline

Fernández C, Goldberg JM (1976b) Physiology of peripheral neurons innervating otolith organs of the squirrel monkey. III. Response dynamics. J Neurophysiol 39:996-1008. Medline

Goldberg JM (2000) Afferent diversity and the organization of central vestibular pathways. Exp Brain Res 130:277-297. CrossRef Medline

Goldberg JM, Smith CE, Fernández C (1984) Relation between discharge regularity and responses to externally applied galvanic currents in vestibular nerve afferents of the squirrel monkey. J Neurophysiol 51:12361256. Medline 
Goldberg JM, Desmadryl G, Baird RA, Fernández C (1990) The vestibular nerve of the chinchilla. IV. Discharge properties of utricular afferents. J Neurophysiol 63:781-790. Medline

Gordon N, Shackleton TM, Palmer AR, Nelken I (2008) Responses of neurons in the inferior colliculus to binaural disparities: insights from the use of Fisher information and mutual information. J Neurosci Methods 169: 391-404. CrossRef Medline

Green DM, Swets JA (1966) Signal detection theory and psychophysics. New York: Wiley.

Gu Y, Fetsch CR, Adeyemo B, Deangelis GC, Angelaki DE (2010) Decoding of MSTd population activity accounts for variations in the precision of heading perception. Neuron 66:596-609. CrossRef Medline

Haburcakova C, Lewis RF, Merfeld DM (2012) Frequency dependence of vestibuloocular reflex thresholds. J Neurophysiol 107:973-983. CrossRef Medline

Jamali M, Sadeghi SG, Cullen KE (2009) Response of vestibular nerve afferents innervating utricle and saccule during passive and active translations. J Neurophysiol 101:141-149. Medline

Jones GM, Young LR (1978) Subjective detection of vertical acceleration: a velocity-dependent response? Acta Otolaryngol 85:45-53. CrossRef Medline

Kalluri R, Xue J, Eatock RA (2010) Ion channels set spike timing regularity of mammalian vestibular afferent neurons. J Neurophysiol 104:2034-2051. CrossRef Medline

Kiang NY (1965) Discharge patterns of single fibers in the cat's auditory nerve. Cambridge, MA: Massachusetts Institute of Technology.

Lansberg MP, Guedry FE Jr, Graybiel A (1964) The effect of changing resultant linear acceleration relative to the subject on nystagus generated by angular acceleration. Aeromed Acta 9:97-122. Medline

Lehmann EL (1983) Theory of point estimation. New York: Wiley.

Lisberger SG, Pavelko TA (1986) Vestibular signals carried by pathways subserving plasticity of the vestibulo-ocular reflex in monkeys. J Neurosci 6:346-354. Medline

MacNeilage PR, Turner AH, Angelaki DE (2010a) Canal-otolith interactions and detection thresholds of linear and angular components during curved-path self-motion. J Neurophysiol 104:765-773. CrossRef Medline

MacNeilage PR, Banks MS, DeAngelis GC, Angelaki DE (2010b) Vestibular heading discrimination and sensitivity to linear acceleration in head and world coordinates. J Neurosci 30:9084-9094. CrossRef Medline

Massot C, Chacron MJ, Cullen KE (2011) Information transmission and detection thresholds in the vestibular nuclei: single neurons vs. population encoding. J Neurophysiol 105:1798-1814. CrossRef Medline

McDonald JJ, Teder-Sälejärvi WA, Hillyard SA (2000) Involuntary orienting to sound improves visual perception. Nature 407:906-908. CrossRef Medline

McDonnell MD, Ward LM (2011) The benefits of noise in neural systems: bridging theory and experiment. Nat Rev Neurosci 12:415-426. CrossRef Medline

Merfeld DM (2011) Signal detection theory and vestibular thresholds. I. Basic theory and practical considerations. Exp Brain Res 210:389-405. CrossRef Medline

Merfeld DM, Young LR (1995) The vestibulo-ocular reflex of the squir- rel monkey during eccentric rotation and roll tilt. Exp Brain Res 106: 111-122. Medline

Merfeld DM, Zupan LH (2002) Neural processing of gravitoinertial cues in humans. III. Modeling tilt and translation responses. J Neurophysiol 87: 819-833. Medline

Merfeld DM, Zupan LH, Gifford CA (2001) Neural processing of gravitoinertial cues in humans. II. Influence of the semicircular canals during eccentric rotation. J Neurophysiol 85:1648-1660. Medline

Naseri AR, Grant PR (2012) Human discrimination of translational accelerations. Exp Brain Res 218:455-464. CrossRef Medline

Pelli DG (1985) Uncertainty explains many aspects of visual contrast detection and discrimination. J Opt Soc Am A 2:1508-1532. CrossRef Medline

Pfingst BE, Xu L (2004) Across-site variation in detection thresholds and maximum comfortable loudness levels for cochlear implants. J Assoc Res Otolaryngol 5:11-24. CrossRef Medline

Purcell IM, Newlands SD, Perachio AA (2003) Responses of gerbil utricular afferents to translational motion. Exp Brain Res 152:317-322. CrossRef Medline

Sadeghi SG, Chacron MJ, Taylor MC, Cullen KE (2007) Neural variability, detection thresholds, and information transmission in the vestibular system. J Neurosci 27:771-781. CrossRef Medline

Seung HS, Sompolinsky H (1993) Simple models for reading neuronal population codes. Proc Natl Acad Sci U S A 90:10749-10753. CrossRef Medline

Shadlen MN, Britten KH, Newsome WT, Movshon JA (1996) A computational analysis of the relationship between neuronal and behavioral responses to visual motion. J Neurosci 16:1486-1510. Medline

Shams L, Kamitani Y, Shimojo S (2000) Illusions. What you see is what you hear. Nature 408:788. CrossRef Medline

Shams L, Kamitani Y, Shimojo S (2002) Visual illusion induced by sound. Brain Res Cogn Brain Res 14:147-152. CrossRef Medline

Smith CE, Goldberg JM (1986) A stochastic afterhyperpolarization model of repetitive activity in vestibular afferents. Biol Cybern 54:41-51. CrossRef Medline

Stein RB, Gossen ER, Jones KE (2005) Neuronal variability: noise or part of the signal? Nat Rev Neurosci 6:389-397. CrossRef Medline

Tollin DJ, Koka K, Tsai JJ (2008) Interaural level difference discrimination thresholds for single neurons in the lateral superior olive. J Neurosci 28:4848-4860. CrossRef Medline

Valko Y, Lewis RF, Priesol AJ, Merfeld DM (2012) Vestibular labyrinth contributions to human whole-body motion discrimination. J Neurosci 32: 13537-13542. CrossRef Medline

Walsh EG (1961) Role of the vestibular apparatus in the perception of motion on a parallel swing. J Physiol 155:506-513. Medline

Walsh EG (1962) The perception of rhythmically repeated linear motion in the horizontal plane. Br J Psychol 53:439-445. CrossRef Medline

Young LR, Meiry JL (1968) A revised dynamic otolith model. Aerosp Med 39:606-608. Medline

Yu XJ, Dickman JD, Angelaki DE (2012) Detection thresholds of macaque otolith afferents. J Neurosci 32:8306-8316. CrossRef Medline

Zupan LH, Merfeld DM (2008) Interaural self-motion linear velocity thresholds are shifted by roll vection. Exp Brain Res 191:505-511. CrossRef Medline 